\title{
Teledentistry; Escalating Horizon in COVID-19
}

\section{Chahal $\mathrm{H}$ and Kaur I*}

BDS, Panjab University, India

*Corresponding author: Ishdeep Kaur, BDS, Dr Harvansh Singh Judge Institute of Dental Sciences and Hospital, Panjab University, India, Email: deptomr@gmail.com

\section{Review Article \\ Volume 5 Issue 4}

Received Date: November 16, 2020

Published Date: December 02, 2020

DOI: $10.23880 /$ oajds-16000281

\section{Abstract}

Teledentistry has emerged as an efficacious technique of extending oral care services to the patients remotely assisted by the sophisticated technology of telecommunication. Over time, teledentistry has established itself as a convenient, labour saving, time effective and an economical alternative alongside bridging the inequality of the available services between the urban and rural demographics. This review explores the evolution and applications of teledentistry and how it has been instrumental in attending to the needs of patients specifically in times of a roaring pandemic where inpatient examination and treatment in a dental facility was not feasible or stalled as a precautionary measure. Not only does it assists in attending to emergencies and routine consults but also in screening and reporting of any plausible cases with COVID infection.

Keywords: Teledentistry; COVID 19; Emergency; Telecommunication

Abbreviations: ISDN: Integrated Services Digital Network; POTS: Plain Old Telephone System; VDH: Virtual Dental Home; CAD: Computer Aided.

\section{Introduction}

Amelioration of the care offered to the patients over time has gained greater dimensions because of continual advancements in technology, methodology and equipment. Telemedicine has revolutionised patient care by communicating with patients remotely, assisted by telecommunication technology. Evolution of internet services facilitated by hardware and software technology has aided telemedicine to its present eminence. Application of telemedicine becomes even more instrumental in providing consultations and necessary care when the patients are refrained from physically accessing health care facilities. Providing dental care services via telecommunication technology constitutes "Teledentistry". Cook in 1997, defined Teledentistry as "the practice of using video-conferencing technologies to diagnose and provide advice about treatment over a distance" [1].

\section{History}

Teledentistry developed as a subspecialist field of telemedicine. A blueprint for dental informatics in 1989 funded by the Westinghouse electronics system group in Baltimore, formed the initial concept of teledentistry [1]. In 1994 United States Army's Total Dental Access Project aimed at improving dental awareness through education and patient care. The project signified enhanced care prospects for patients with reduced costs [1].

\section{Development of Teledentistry Over the Time}

Enhancement in the telecommunication services over time in terms of speed and mechanism of data transfer has transformed teledentistry to an indispensable asset.

\section{Image File Transfer through Modem}

Dental image management systems were used concurrently with intra-oral cameras in a study at Georgia to generate patient's intra-oral images, which were later relayed via a modem between dental clinics 120 miles apart. 
Fifteen patients who had undergone periodontal surgeries in one and were under the post-operative care with the other clinic, were affirmative of finer experience at treatment, as the status of their healing was shared between both the clinics via images, saving time and travel [2].

\section{File Image Transfer through Satellite}

A study performed in 1995 in Haiti used a satellite over international maritime for video teleconferencing for consultation between deployed dentists and specialists at army medical centre. It depicted that while video quality wasn't conducive of diagnosis, images captured were of diagnostic importance [2].

\section{Integrated Services Digital Network (ISDN)}

ISDN and video teleconferencing were used by army posts to relay images, radiographs for video consultation to medical centres for support in diagnosis and treatment planning. ISDN system proved valuable but stumbled because of the high cost of equipment and its maintenance [2].

\section{Plain Old Telephone System (POTS)}

Teledentistry network based on POTS in 1997 was used in smaller clinics in parts of Europe, which consisted of a computer and its components and an intra-oral camera to collect patient information for an online database including the frequency of consults delivered, the satisfaction of patients and dentists using the network and discrepancies experienced with the network which stood as a source of analyses [2].

\section{Web-Based System}

The web-based system was facilitated by internet services, where web browser was used in computers and laptops along with intraoral cameras to relay data amongst dentists, interdisciplinary specialists and laboratories with all the information stored on a database. This system has been on a constant enhancement with the introduction of features of $3 \mathrm{D}$ representation enabling modifications to orientation and image characteristics [2].

\section{Forms of Telecommunication}

Teledentistry is practised in two variants.

\section{Real-Time/Synchronous Consultations}

It involves video consultations between the dentist and the patients via telecommunications where the interaction between the two is streamed live and the dentist provides the patient with immediate consult [1].

\section{Store and Forward/Asynchronous}

All information of the patient is stored, batched, assigned and triaged on a web-based database to be analysed by the dentist later in time. Accordingly, the dentist interprets the data and formulates an appropriate diagnosis and treatment plan. The database could also be effectively used to relay data using secure portals in cases where interdisciplinary treatment is involved [1].

\section{Application in Dental Specialities}

\section{Oral and Maxillofacial Surgery}

Extensive surgeries involving prosthetic constructions could be observed by the specialists around the world along with providing their assistance to the procedure with their approach and inputs. Rollert MK, et al. demonstrated that the pre-operative assessment aided by telecommunication was an effective alternative for patients scheduled for dentoalveolar surgery, specifically in cases where patient transport is not feasible [3]. A study by Duka M, et al. to investigate the efficacy of teledentistry in the diagnosis of impacted third molars depicted the method to be equally effective as a diagnosis made by clinical assessment [4].

\section{Radiology and Oral Medicine}

Transfer of intraoral and panoramic radiographs, computed tomography, magnetic resonance imaging and cone-beam computed tomography for specialist consult and review. Tele-radiology could hence assist in reporting rapid and accurate diagnosis. Electronically photographed oral lesions along with the clinical presentation of the associated symptoms are analysed by specialists of oral medicine for remote consultations. Bradley $\mathrm{M}$, et al. demonstrated the efficacy of oral medicine in community service in Ireland using teledentistry [5]. Torres Pereira C, et al. evaluated diagnosis in oral medicine through the transfer of clinical images digitally via email [6].

\section{Orthodontics}

Impressions made can be digitalised into threedimensional models. The web-based applications can stimulate the functions of the orthodontic appliances and the oral musculature on the models. The specialist can use the 3D models for consultations with his fellow dentists and in explaining the course of the treatment to the patient. Orthodontic emergencies arising from the uneventful breakage of ligature wires and other appliances can be dealt with at priority. The specialist can guide the patient through, 


\section{Open Access Journal of Dental Sciences}

with all necessary steps to help prevent any immediate damage from the breakage. Berndt $F$, et al. reached an inference that interceptive orthodontic treatment by general dentist assisted by orthodontists through teledentistry was suitable in intercepting the developing malocclusion, in otherwise underserved children [7]. Favero, et al. concluded after studying tele-assistance method of communication, that transfer of images and videos was effective to resolve minor complication at the patient's end, reassuring parents and saving time on visiting the dentist [8].

\section{Public Health Dentistry}

Community dental health programs at schools, rural and remote areas via regular educative and screening programs aided by telecommunication. The Virtual Dental Home System of Care (VDH) is a community based oral health delivery system of University of Pacific, in which people receive dental diagnostic, preventive and early intervention services in a community setting. A dental hygiene model developed by Northern Arizona University Dental Hygiene Department was reported by Summer felt FF to be serving the underprivileged by collaborating with experts remotely.

\section{Pediatric and Preventive Dentistry}

Pediatric patients can be made acquainted with the dentist and the clinic set up over video consultation, well beforehand the actual visit to the clinic which proves beneficial to reduce the fear associated with the visit later. Not only does teledentistry assist in early interception of diseases such as dental caries but also it acts as a tool to impart awareness among patients concerning oral hygiene practices and habits. Purohit, et al. assessed the reliability of video graphic tools to screen dental caries in school going children and concluded that teledentistry to be an effective tool in screening and assessment. Kopycka-Kedzierawski DT and Billings RJ carried out a study examining the innovative screening modalities which were less labour intensive, costeffective and had the potential to identify children with a high risk of caries [9].

\section{Prosthodontics}

Communication between the dentist and the technician at the laboratory concerning the design, size, shape of the prosthesis and shade selection of teeth [3]. Computerized dentistry specialists can assist the technicians using telecommunications in using computer-aided manufacturing systems (CAD) in designing the dental prosthesis. A study by Ignatius $\mathrm{E}$, et al. depicted the potential use of video consults in delivering specialist services in scattered populations requiring oral rehabilitation treatment [10].

\section{Forensic Dentistry}

Digitalised models can be used in surveys, studies and in solving criminal cases.

\section{Benefits of Teledentistry}

\section{Emergency care}

Collaboration with local urgent care centres enables immediate consultation and assistance in providing emergency supportive treatment in cases of extensive damage to oro-facial structures. The dental specialist can guide the patient through with the necessary measures when contacted in cases of tooth fracture or breakage of a dental prosthesis. Reassurance from the dentist would prevent the patient from developing hysteria.

\section{Timely Diagnosis and Intervention}

Most individuals including children typically receive dental care after they have developed the advanced disease, pain and infection which can be all prevented with regular screenings and early interception of any developing disease.

\section{Saves On Time and Resources}

Video consultations save the time spent on travelling the distance to the dentist. Easy access to web-based applications and secure web portals in establishing a virtual connection of the patient with the dentist has established tele-consults to be economical and user friendly.

\section{Efficient Relay of Information}

Data including the previous treatment history, charts, clinical images, diagnostic radiographs, three-dimensional models, prosthesis designs along with the test results can be relayed to other specialists, dental technicians and laboratories via secure remote access software.

\section{Education/Training}

Teledentistry could effectively benefit virtual study clubs in case evaluations and interdisciplinary dental education, delivering lectures via teleconferencing, broadcasting videos and courses for continuing education programs.

\section{Teledentistry and its use during COVID-19 pandemic}

Teledentistry uses information technology to provide education, guidance or treatment to patients [11]. Teledentistry is a subunit of telehealth along with 


\section{Open Access Journal of Dental Sciences}

telemedicine [12]. It is used for real-time consultation, diagnosis and treatment planning in areas of deficiency of facilities, in schools, remote areas and long-term healthcare facilities $[12,13]$. In today's time of the pandemic, the main focus is to avoid direct face-to-face contact [12]. Teledentistry satisfies the need for social distancing required all around the world to contain the spread of SARS-COV-2 virus as 'Tele' means 'distant' [12].

\section{Patient Management by Teledentistry}

Telephone screening all patients for symptoms of COVID-19 is immensely important. Dentists are required to use the Phone advice Line Tool for Possible COVID-19 patients as recommended by the CDC [14]. Teledentistry is playing a vast role in detecting the suspected COVID-19 patients and at the same time, it also decreases the exposure of infected patients into the dental office and hospitals [12]. If patients show symptoms then delay in the dental office treatment should be made until the patient recovers and the patient should be treated through phone appointments rather than in-office appointments [14].

\section{Screening for COVID-19 Symptoms through Teledentistry}

Teledentistry is playing a vast role in detecting the suspected COVID-19 patients and at the same time, it also decreases the exposure of infected patients into the dental office and hospitals $[15,12]$. Some recommendations made by the CDC are pre-screening questions regarding COVID-19.

\section{Special Circumstances}

a) Ask "Do you/they live in a nursing home or other longterm care facility?"

b) "In the last two weeks have you worked or volunteered in a hospital, emergency room, clinic, medical office, ambulance service, first responder services, or any health care setting, or taken care of patients as a student as a part of your work?"

\section{Potential Challenges of Teledentistry}

Diagnosis made by teledentistry cannot be as accurate as performed clinically by palpation and percussion. Teledentistry, however, is only helpful for preventive and diagnostic purposes [11]. Only medical prescriptions for the pain or antibiotics can be given through teledentistry but, for specific clinical treatment such as restoration and surgeries patients have to visit the dental office [11]. Teledentistry might be technologically challenging (connectivity issues) for the dentist and might have limitations to technological devices such as; smartphone, tablet, computer $[12,16]$.

\section{Challenges Faced by Dentistry Related to Acceptance to Teledentistry}

Dentists also might be complex and resistant to new skills [12]. Dentists are usually afraid of making an inaccurate diagnosis [12]. There can also be limitations regarding infrastructure such as poor internet access, lack of technical skills, hardware shortage, lack of training [12]. Regulatory issues as well as interstate licensure challenges may take place [16]. Dentists have to make clinical judgement and diagnosis based on two-dimensional photographs or video recording which may not be clear as the photographs may have low quality. Technical problems may also occur during data transmission and may cause misdiagnosis or medical error [11]. Telehealth is disruptive, complex and requires clinicians to learn new methods of consulting [15]. Consideration of malpractice should also be made in such cases. This places the dentist in a difficult situation. Moreover, depending on the health condition and urgency situations the patient might need emergency treatment [16].

\section{Challenges Faced by Patients Related to Acceptance of Teledentistry}

Cultural acceptance to teledentistry also plays a vital role in the limitation or success of telehealth. The patient might be uncomfortable on virtual appointments and might also be concerned about privacy [16]. Therefore, informed consent should be taken from the patient and the patient should be aware of the inherent risk of improper diagnosis or treatment due to the failure of technology [11]. Patients also might not be able to communicate and apprehend their problem properly. Most of the teledentistry is in English. Since internet technology in English, the future goal should include considering other languages in the program as well [11]. Acceptance of teledentistry will only increase as the acceptance of telemedicine is increased [12]. Furthermore, the initial cost of equipment is extremely high, such as; intraoral digital camera and high-speed internet for virtual appointments is required [11]. Surveys have also found that teledentistry is gaining acceptance and appreciation by patients as well as healthcare providers especially during the time of pandemic [11].

\section{Recommendations to Overcome the Challenges}

These challenges need to be overcome by training and educating the dentist about technology [12]. Dentists should be skilled, capable and efficient at work as well. Also, the dental school curriculum should be updated according to the teledentistry measurements. This will send a clear message to current and future healthcare professionals that telehealth 
is a legitimate part of usual care [15]. Care of documentation should be done by the respective dentist to reduce any liabilities. Organized record of emails, consent forms, patients medical records and charts should be kept by the practitioner in charge in the office or hospital. Also, the dentist needs to clarify regarding the installation, maintenance, access, security, and privacy efforts associated with the equipment used before proceeding with teledentistry [16]. Standard clinical guidelines according to the CDC should be maintained and accepted for better dental care in the dental community [16].

\section{Conclusion}

Teledentistry is a vast growing aspect of dentistry especially during this uncertain time of Covid-19. Even though there are many challenges related to acceptance of teledentistry by both the dentist as well as the patient; it satisfies the need for social distancing required to decrease the spread of SARS-COV-2 virus. Moreover, it offers patient care at a reasonable cost. As recommended by the CDC, screening for SARS-COV-2 virus can be done before dental appointments. Dental lab communications as well as longdistance clinical training is also being accepted through telecommunication. The application of teledentistry is of great importance especially to rural areas where there is a lack of specialists and lack of sophisticated healthcare systems. In future teledentistry, if adequately funded is going to be the requirement as well as the necessity of the healthcare industry.

\section{References}

1. Jampani ND, Nutalapati R, Dontula BS, Boyapati R (2011) Applications of teledentistry: A literature review and update. J Int Soc Prev Community Dent 1(2): 37-44.

2. Bhargava A, Sabbarwal B, Jaggi A, Chand S, Tandon S (2019) Teledentistry: A literature review of evolution and ethicolegal aspects. J Global Oral Health 2(2): 128133.

3. Rollert MK, Strauss RA, Abubaker AO, Hampton C (1999) Telemedicine consultations in oral and maxillofacial surgery. J Oral Maxillofac Surg 57(2): 136-138.

4. Duka M, Mihailovic B, Miladinovic M, Jankovic A, Vujicic B (2009) Evaluation of telemedicine systems for impacted third molars diagnosis. Vojnosanit Pregl 66(12): 985991.
5. Bradley M, Black P, Noble S, Thompson R, Lamey PJ (2010) Application of teledentistry in oral medicine in a community dental service, N. Ireland. Br Dent J 209(8): 399-404.

6. Torres Pereira C, Possebon RS, Simões A, Bortoluzzi MC, Leão JC, et al. (2008) Email for distance diagnosis of oral diseases: a preliminary study of teledentistry. J Telemed Telecare 14(8): 435-438.

7. Berndt J, Leone P, King G (2008) Using teledentistry to provide interceptive orthodontic services to disadvantaged children. Am J Orthod Dentofacial Orthop 134(5): 700-706.

8. Favero L, Pavan L, Arreghini A (2009) Communication through telemedicine: home teleassistance in orthodontics. Eur J Paediatr Dent 10(4): 163-167.

9. Kopycka Kedzierawski DT, Billings RJ, McConnochie KM (2007) Dental screening of preschool children using teledentistry: a feasibility study. Pediatr Dent 29(3): 209-213.

10. Ignatius E, Perälä S, Mäkelä K (2010) Use of videoconferencing for consultation in dental prosthetics and oral rehabilitation. J Telemed Telecare 16(8): 467470.

11. Arora PC, Kaur J, Kaur J, Arora A (2019) Teledentistry: An innovative tool for the underserved population. Digit Med 5(1): 6-12.

12. Ghai S (2020) Teledentistry during COVID-19 pandemic. Diabetes Metab Syndr 14(5): 933-935.

13. Alabdullah JH, Daniel SJ (2018) A Systematic Review on the Validity of Teledentistry. Telemed J Health 24(8): 639-648.

14. Sfikas PM (1997) Teledentistry: Legal and regulatory issues explored. J Am Dent Assoc 128(12): 1716-1718.

15. Smith AC, Thomas E, Snoswell CL, Haydon H, Mehrotra A, et al. (2020) Telehealth for global emergencies: Implications for coronavirus disease 2019 (COVID-19). J Telemed Telecare 26(5): 309-313.

16. Rocca MA, Kudryk VL, Pajak JC, Morris T (1999) The evolution of a teledentistry system within the Department of Defense. Proc AMIA Symp pp: 921-994. 